\title{
ALUMINUM COATING IN THE UNDULATOR VACUUM CHAMBER FOR THE LINAC COHERENCE LIGHT SOURCE*
}

\author{
Dean Walters ${ }^{\#}$ \\ Advanced Photon Source, Argonne National Laboratory, Argonne IL 60439, USA
}

\begin{abstract}
A prototype vacuum chamber is under development at the Advanced Photon Source that is intended for use in the Linac Coherent Light Source at Stanford Linear Accelerator Center. The chamber will be fabricated from the austenite stainless steels. The chamber requires a continuous aluminum coating on the inner surface in order to reduce the wakefield losses to a level within the resistivity budget. The method being presented here is unique in that it can be applied to a fully fabricated chamber $5 \mathrm{~mm}$ high, $12.5 \mathrm{~mm}$ wide, and $3460 \mathrm{~mm}$ long. In existing methods the chamber aperture has been much larger than is used here. This paper describes a method applicable for these smaller cross sections. This process uses a pair of small electrodes, centered in the aperture, where they are attached to a high-frequency AC power supply. In this configuration each electrode is connected to the opposite polarity of the other. The chamber cavity is filled with argon gas to facilitate the formation of a glow discharge causing the aluminum electrodes to sputter onto the chamber walls. This paper presents the laboratory test results from small samples up to the fullsized assemblies.
\end{abstract}

\section{INTRODUCTION}

The Linac Coherent Light Source (LCLS) will be the world's first x-ray free-electron laser when it becomes operational in 2009. The LCLS is a US-DOE-funded project that is currently in the detailed project engineering phase with many sections already fabricated and installed.

The portion of the accelerator line that produces x-rays is the undulators. The 33 undulator assemblies are the heart of the LCLS free-electron laser with a total length measuring 130 meters long. Within the undulator is the vacuum chamber through which the electron beam will travel.

\section{DESCRIPTION OF CHAMBER}

The vacuum chamber is made up of the strongback, the end cap, and the two side walls (see Figure 1). The aperture through the chamber is narrow, $5 \mathrm{~mm} \times 12.5 \mathrm{~mm}$, and long, 3.5 meter, made in a stainless steel construction where the inner surface is highly polished and coated with aluminum.

The support strongback is made of $20 \mathrm{Cb}-3$, also known as Carpenter Alloy 20, which is an austenitic stainless steel alloy that has proven to have a low magnetic

*Work supported by U.S. Department of Energy, Office of Science, under Contract Nos. DE-AC-02-06CH11357 and DE-AC02-76SF00515.

\#drw@aps.anl.gov permeability even after it has been welded [1,2]. The

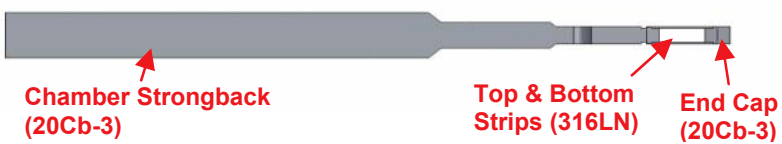

Figure 1: Chamber cross section.

inside face of the strongback is polished to a surface finish better than $0.1 \mu \mathrm{m}$. An end cap is made of the same alloy and its inside surface is also is polished to $0.1 \mu \mathrm{m}$. The side walls are made from sheets of type- 316LN stainless steel with an inner face CMP- polished to a surface finish better than $0.05 \mu \mathrm{m}$. All these parts are carefully laser welded and processed in the Vacuum Group shop at Argonne National Laboratory. An internal aluminum coating of minimum thickness $0.1 \mu \mathrm{m}$ is applied before it is baked out.

\section{CONFIGURATION OF COATING SYSTEM}

The coating process used here is a dual-cathode AC diode sputtering [3] configuration as shown in the schematic in Figure 2. The apparatus is made up of two aluminum tubes that are suspended through the middle of the chamber aperture. The tubes have flowing water for cooling and are vacuum sealed and electrically isolated using rf hollow conductor feedthroughs. Lowconductivity water is circulated by a process chiller that is used to keep the temperature of the cathodes under closed- loop control. Power to the cathodes is supplied by a mid-frequency rf power supply. The chamber and the attached end stations are pumped using a dry multi-stage Roots-type pump with a pumping speed of $16 \mathrm{cfm}$. The flow-controlled gas inlet system uses a mass flow controller to meter the argon flow into the system.

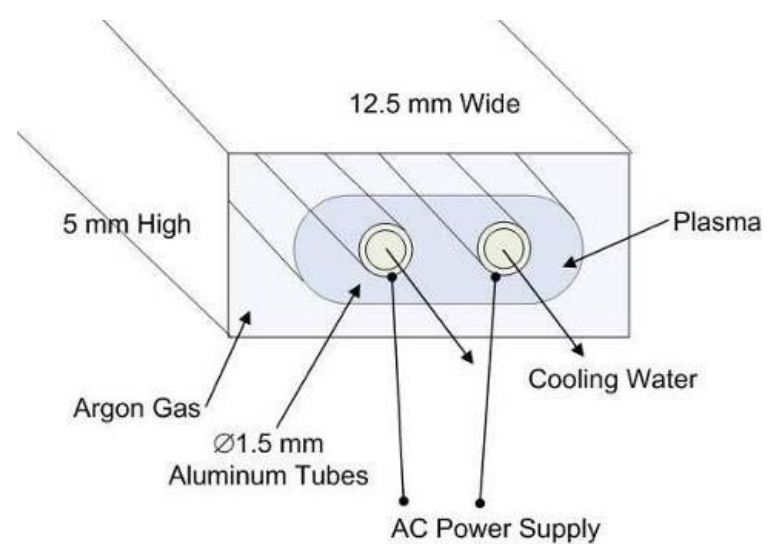

Figure 2: Basic sputtering setup. 
The theory of operation is that after pumping the system down to $5 \mathrm{mTorr}$, the system is filled to 1.2 Torr with argon gas. After running an argon gas purge for some length of time, the power supply is turned on and at low power, 60 watts in this case, the gas is ionized into a glow discharge. Upon increasing the power to 800 watts, deposition of the aluminum starts. In this configuration each cathode is connected to one side of the power supply and the chamber walls are grounded. So a glow discharge forms around the two cathodes, and its shape is controlled by the cathode-to-wall distance and the argon pressure. The polarity of each cathode is changing at a frequency of $50 \mathrm{kHz}$, which is a distinct advantage with the aluminum oxide that is initially covering the cathodes. Aluminum oxide is a dielectric that will charge up with electrons during the negative portion of the cycle. When the polarity switches to positive, the electrons will be discharged thus avoiding a damaging arc that can ruin the polished chamber surface and the aluminum coating. As the process progresses, the thin aluminum oxide coating will be removed and pure aluminum will then be sputtering from the surface. The aluminum is sputtered off the negative cathode by the accelerating argon ions colliding into it and ejecting aluminum. The aluminum will travel from the cathode until it strikes a surface and adheres to it. Since this process is performed at a high pressure, $\sim 0.7$ Torr, the distance that the aluminum can reach is limited to $\sim 30 \mathrm{~mm}$ due to the short mean-free path. With chamber walls only $<5 \mathrm{~mm}$ away, there is no lack of coverage.

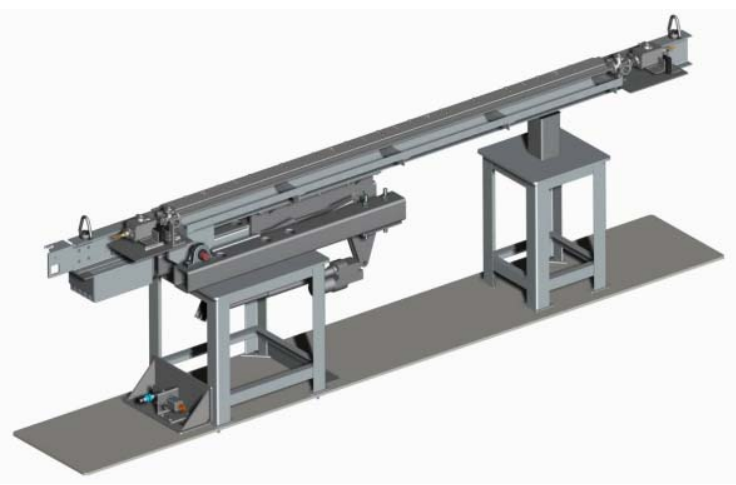

Figure 3: Coating fixture.

\section{SYSTEM FOR COATING FULL-SIZED CHAMBERS}

A coating fixture was constructed for the purpose of mounting the chamber and affixing the cathodes into place. Figure 3 shows the three parts that comprise the crating fixture: chamber mounting platform, elevation mechanism, and end stations.

The chamber platform is made of an I-beam for bolting down the chamber. The chamber will have a lengthwise bow in it, and the machined flat I-beam constrains it to a straight condition.

The elevation mechanism (see the actuator under the left table) has the ability to swing the chamber platform up to a vertical position. This was in anticipation of the 14- foot cathodes contacting the chamber walls. At this point in the development it has not been needed since the tensioning of the cathodes has not required it.

The end stations entail the clamping of the cathodes, the gas inlet, vacuum outlet, cathode positioning micrometers, and $\mathrm{rf}$ feedthroughs. The end stations are a considerable improvement over the bench test set up in that the spring loading of the cathodes has mitigated the problem of the cathodes contacting the side walls.

\section{RESULTS OF THE THIN FILM PROCESS DEVELOPMENT}

The chamber coating fixture was used to both coat chambers and to run process development tests. When chambers were not being coated, spools were substituted for tests. Figure 4 shows the system with the stainless steel and glass spools in place. Tests were initially run with a $\varnothing 1.5 "$ tube, and then all later tests were run with a 0.5 "- ID tube to better approximate the conditions of the small- aperture vacuum chamber. Inserting $\mathrm{Si}$ samples into the tubes and measuring the film thickness using a profilometer allowed us to measure a number of parameters of the coating process. One of the first was to establish the film thickness uniformity along the length. Three different coating runs using samples mounted at four points down the 0.5 "-ID tubes established a uniformity ranging from $4.3 \%$ to $11.6 \%$.

A quartz crystal deposition monitor was installed into the middle, and the sensor was positioned $10.3 \mathrm{~mm}$ from the cathodes. These results were scaled with thickness measured from Si samples that were placed in the 0.5"ID tubes; see data shown in Figure 5. The three runs demonstrate the effect of start-up conditions on the deposition process where all were run at the same process pressure, 0.715 Torr, and the same power setting, 1200 watts. In Run $\# 1$, using a pre-conditioned cathode, the argon purge was limited to one hour, so in the beginning the glow discharge was scrubbing the system and the deposition was negative. At 30 minutes into the run the system was clean enough to start to build up a film. In Run \#2 the purge was five hours long, and the clean system responded by starting to build up a measurable film at 820 watts. The rate rapidly increased as the power was incremented to 1,000 watts and then to 1,200 watts. In Run \#3 the cathodes were replaced and the system was purged for six hours. While there was no evidence of scrubbing, the deposition was reduced due to the lack of conditioning of the cathodes.

Figure 6 is a result of calculating the deposition rate from the raw data that was presented in Figure 5. This figure includes a curve of the input power for both the conditioned and the cathode in the initial state. The conditioned cathode has a much higher deposition rate than the unconditioned cathode. A visual examination shows that the conditioned cathode has a matte finish while the raw aluminum tubing has a bright luster. 


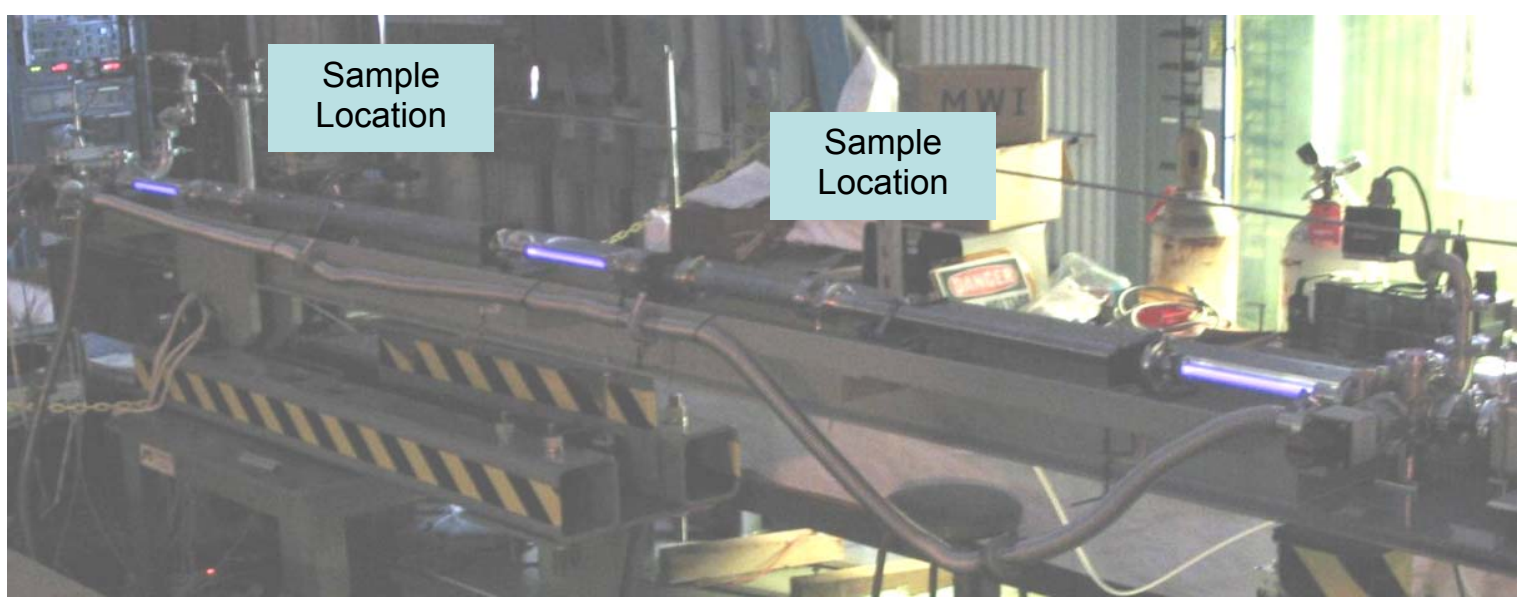

Figure 4: Glow discharge seen through glass spools.

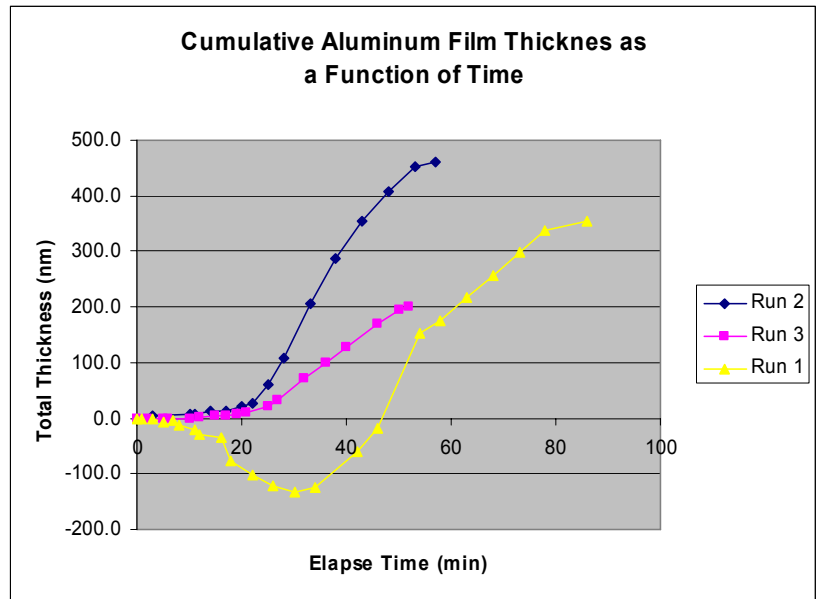

Figure 5: Cumulative deposition curves.

To better understand the output of the power supply, an oscilloscope was connected to the power leads on the rf feedthrough. The voltage was measured at various power settings for a period of 3-5 minutes. It was found that at a power setting of 1400 watts the voltage, rms, is 1240 volts.

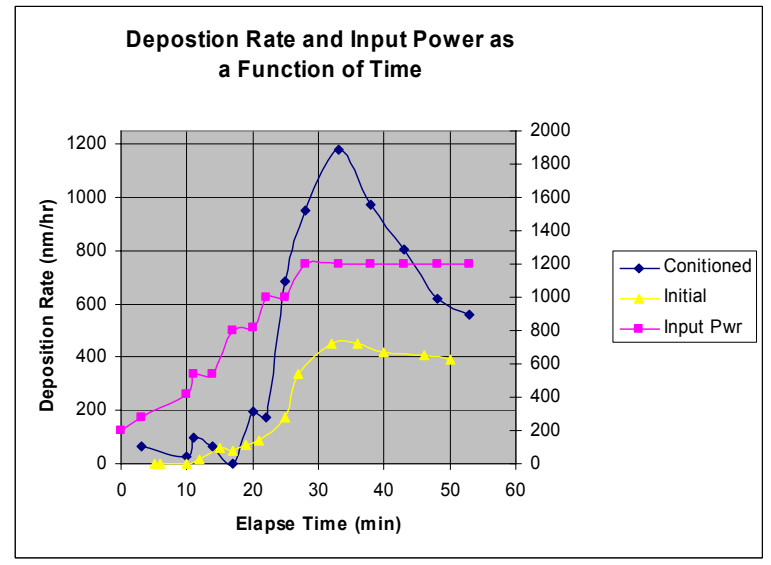

Figure 6: Deposition rate curves.

\section{CONCLUSIONS}

This new sputtering technique has been shown to be effective in the internal coating of the 5-mm-high LCLS undulator vacuum chamber (see Figure 7). The results to date show that the aluminum sputters evenly down the chamber length. They also demonstrate that cathode conditioning and purge time do have an effect on deposition rate. The drop of deposition rate under constant power is an interesting result and will be pursued in future work.

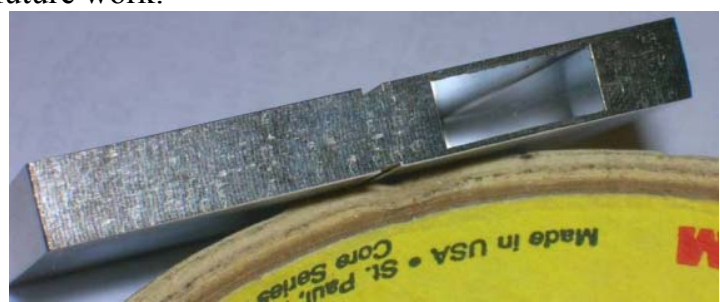

Figure 7: Coated chamber.

\section{ACKNOWLEDGMENTS}

The guidance of Grant Este played a major factor in all of this work. The help and cooperation of Geoff Pile, Stephen Milton, Guy Harris, Darrell S. Doran, Soon-Hong Lee, Frank Depaola, James Bailey, and James Morgan were central in getting this system put together under the pressure of the project.

\section{REFERENCES}

[1] S.H. Lee et al., "Magnetic Properties of the Undulator Vacuum Chamber Materials for the Linac Coherent Light Source," Proceedings of the 27th International Free Electron Laser Conference, 21-26 August 2005, Stanford, California, 383-386 (2005).

[2] N. Wilson and P. Bunch, "Magnetic Permeability of Stainless Steel for Use in Accelerator Beam Transport systems," PAC1991, 2322, http://www.jacow.org.

[3] G. Este and W.D. Westwood, "A quasi-direct-current sputtering technique for the deposition of dielectrics at enhanced rates," J. Vac. Sci. Technol. A 6(3), 1845 (1988). 